\title{
Um modelo computacional baseado em Redes Neurais Artificiais para auxiliar o reconhecimento de cores por portadores de Daltonismo
}

\author{
Mergulhão, E. W. T. ${ }^{1}$, Andrade, S. H. M. S. ${ }^{1}$, Nascimento, J. O. do* ${ }^{2}$ \\ * jeffersonascimento@gmail.com, ewtm@msn.com \\ ${ }^{1}$ Faculdade Estácio de Belém, Belém/PA, Brasil. \\ ${ }^{2}$ Programa de Modelagem Computacional, CIMATEC, Salvador/BA, Brasil
}

\begin{abstract}
Resumo
Daltonismo é uma deficiência visual não tão rara, visto que aproximadamente $8 \%$ da população mundial masculina a possui, dificultando o indivíduo na distinção de determinadas cores, de acordo com o tipo de daltonismo. Este trabalho apresenta a construção de um modelo computacional baseado em Redes Neurais cuja função é auxiliar indivíduos daltônicos no seu cotidiano a identificar objetos que possuam alguma cor na qual o ele não consiga distingui-la. Permitirá também que, qualquer cidadão com acesso ao supracitado modelo, possa descobrir se possui ou não daltonismo, de uma maneira rápida e eficiente. Uma rede neural é um processador paralelamente distribuído, constituído de unidades de processamento simples, que tem a propensão natural de armazenar conhecimento experimental e torná-lo disponível para uso, principalmente o de sua capacidade de aprendizagem através de generalização da resposta adquirida durante o treinamento da rede. Utilizamos o serviço IBM Watson Visual Recognition que faz uso de algoritmos de deep learning para analisar imagens, o que resultou em um menor tempo de processamento e o projeto passou a ter um custo financeiro menor. Desta forma, como resultado, construímos um aplicativo mobile cujo acionamento dele ocorre por meio da câmera do smartphone, para o reconhecimento da cor desejada. Por fim, o aplicativo construído, estará disponível para os usuários nas diversas plataformas digitais para download.
\end{abstract}

Palavras-Chave: Modelagem Computacional, Processamento de imagem, Daltonismo, Aplicativo Móvel.

\begin{abstract}
Color blindness is a not so rare visual deficiency, since approximately $8 \%$ of the male world population owns it, making it difficult for the individual to distinguish certain colors according to the type of color blindness. This work presents the construction of a computational model based on Neural Networks whose function is to help color-blind individuals in their daily life to identify objects that have some color in which they can not distinguish it. It will also allow any citizen with access to the aforementioned model to be able to find out whether or not he has color blindness in a fast and efficient way. A neural network is a parallel distributed processor, composed of simple processing units, which has the natural propensity to store experimental knowledge and make it available for use, especially that of its learning capacity through generalization of the response acquired during the training of the network. We used the IBM Watson Visual Recognition service that makes use of deep learning algorithms to analyze images, which resulted in a shorter processing time and the project had a lower financial cost. In this way, as a result, we build a mobile application whose activation of it occurs through the camera of the smartphone, to recognize the desired color. Finally, the application built, will be available to users on the various digital platforms for download.
\end{abstract}

Keywords (Palavras chaves): Keywords: Computational Modeling, Image Processing, Color Blindness, Mobile Application. 


\section{Introdução}

As cores desempenham um papel importante no cotidiano do ser humano, sendo associadas as diversas conotações, cuja aplicação permeia por diversos ramos, desde o uso em semáforos, formas geométricas básicas nas representações de entidades, vestes que caracterizam profissões em local de trabalho como sinalização de segurança que adotam diferentes tipos de cores para cada finalidade e denota uma linguagem universal que não coaduna com idiomas. Entretanto, um em cada dez homens sofre de daltonismo (em mulheres a incidência é pequena). Trata-se da incapacidade de discernir cores, havendo problemas em células nervosas (cones) dos olhos [1].

O problema do reconhecimento de um padrão isolado em uma imagem alvo bidimensional consiste à existência do padrão na imagem, seu grau de escalonamento, ângulo de rotação e posição [2]. Um algoritmo clássico para a classificação de cores, denominado método de imposição de limiares, tem como ideia fundamental determinar paralelepípedos, que envolvam grupos de pixels de uma mesma cor no espaço de cores. Nesse sentido, tal algoritmo mostra-se inadequado para uma série de situações práticas onde os grupos de pixels não são separáveis por paralelepípedos. Assim, novas ferramentas tais como a lógica fuzzy [3] [4] e as redes neurais artificiais [5] têm sido propostas para a tarefa da classificação de cores. Redes Neurais Artificiais podem ser vistas como um sistema adaptativo cujo funcionamento é baseado na forma como o cérebro humano processa as informações e na interação entre seus constituintes fundamentais, os neurônios. São compostas por um conjunto de neurônios artificiais, conectados entre si por meio de sinapses artificiais, representadas por vetores ou matrizes de pesos sinápticos. De forma semelhante ao cérebro humano, o processamento das informações pelas RNA's é distribuído paralelamente pelos neurônios. Além disso, possuem capacidade de aquisição de conhecimento experimental por meio de experiências de aprendizagem [6].

\section{Metodologia}

O presente trabalho foi realizado dentro de um projeto que tem por finalidade a identificação das cores monocromáticas usando redes neurais. A visão de cores é Reconhecimento de Cores Utilizando Redes Neurais Artificiais para Daltônicos um fenômeno complexo, que envolve células fotossensíveis especiais, os cones. Na retina humana, existem aproximadamente cinco milhões de cones e cada um contém um tipo específico de fotopsina: vermelha, verde ou azul. A fotopsina é a proteína responsável por converter o sinal luminoso em sinal elétrico, que é conduzido pelo nervo óptico até o córtex cerebral, onde a visão cromática é interpretada. Cada fotopsina é sensível à luz com um comprimento de onda distinto. Em humanos, o mecanismo de visão de cores é fundamentalmente tricromático, pois as diferentes proporções de estimulação dos três tipos específicos de cones explicam todas as outras cores [7]. A cada dia que passa a busca por ambientes de trabalho mais seguros faz com que os profissionais envolvidos nesta atividade adotem as mais variadas formas de ação fugindo da utilização apenas das formas mais clássicas e básicas de atuação. Isso na prática quer dizer muitas coisas - algumas desta surgem da criatividade e conforme a necessidade da realidade das organizações - outras são, na verdade antigas técnicas de prevenção que vão sendo redescobertas e readequadas aos tempos atuais, que tal processo ocorre de forma extremamente rápida e em condições bastante favoráveis; por exemplo, uma pessoa com acuidade visual normal é capaz de identificar uma letra a uma distância 700 vezes maior do que a altura da mesma (860 vezes a distâncias muito pequenas). Ou seja, uma letra de um centímetro a uma distância de 7 metros, ou letras de $2 \mathrm{~mm}$ a uma distância de 1,40 metro [8].

Apesar dos avanços tecnológicos na área da saúde, inclusive com a utilização de aplicativos móveis, se trata na detecção de indivíduos não-daltônicos, que por sua vez, ainda não se identificaram tais como, visto que aproximadamente $10 \%$ da população masculina no Brasil possui esta deficiência [9]. O uso de aplicativos móveis para auxiliar no cotidiano de indivíduos com alguma deficiência ou de pessoas com alguma doença tem se mostrado uma ferramenta útil e prática na vida dos pacientes. Aplicando isso no contexto do daltonismo, a ferramenta que será desenvolvida no presente trabalho, é caracterizada por um aplicativo móvel, que possa ser utilizado como uma solução de fácil acesso, de auxílio no cotidiano de pessoas com daltonismo, permitindo um diagnóstico de maneira prática ao fornecer uma ferramenta que auxilie aos portadores da deficiência visual, em tempo real, a identificar elementos do mundo real em que possui dificuldade de distinção de suas cores. Este objetivo geral pode ser subdividido em dois objetivos mais específicos: implementar um teste dentro do aplicativo, capaz de diagnosticar o daltonismo do usuário, caso ele possua; utilizar a câmera do dispositivo móvel para identificar todos os objetos do mundo real e as cores em que o usuário, após reconhecer o tipo daquela cor ,saber para qual finalidade vai ser usada diante do cotidiano e as sinalizações na segurança de trabalho.

\section{Fundamentação Teórica}

\subsection{Daltonismo}

Daltonismo, nome mais popular da discromatopsia, é uma deficiência dos cones, localizados no olho, mais precisamente na região central da retina, e que possuem a função de perceber 
as cores [10]. Um indivíduo normal possui três tipos de cones: um responsável por distinguir o vermelho e suas variantes, outro por distinguir a cor verde e suas variantes e um último por distinguir a cor azul e suas variantes, contemplando assim estas 3 cores principais, as quais fazem com que o indivíduo consiga distinguir todas as cores. É, portanto, uma deficiência que dificulta a percepção das cores, fazendo com que o indivíduo daltônico não consiga ou tenha dificuldade de enxergar determinada(s) tonalidade(s) de cor(es), de acordo com o tipo de daltonismo. É, na grande maioria dos casos, hereditária, isto é, é passada geneticamente de pais para filhos e não possui uma cura.

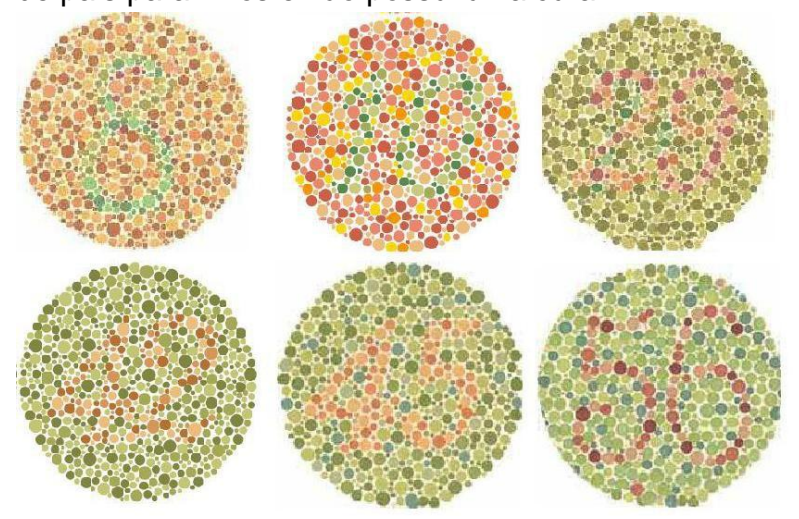

Fig. 1 - Teste de ishirara para descobrir se possui a deficiência.

\subsection{Redes Neurais}

Redes Neurais Artificiais são técnicas de inteligência artificial inspiradas no funcionamento dos neurônios no cérebro humano. É um sistema adaptativo composto por unidades de processamento interconectadas, chamadas de neurônios, distribuídas em diferentes camadas trabalhando em união para a resolução de um problema. A grande vantagem no uso de redes neurais artificiais para solução de problemas complexos provém, principalmente, de sua capacidade de aprendizagem através de generalização da resposta adquirida durante o treinamento da rede, através do uso de exemplos já ocorridos no passado (Figura 2):

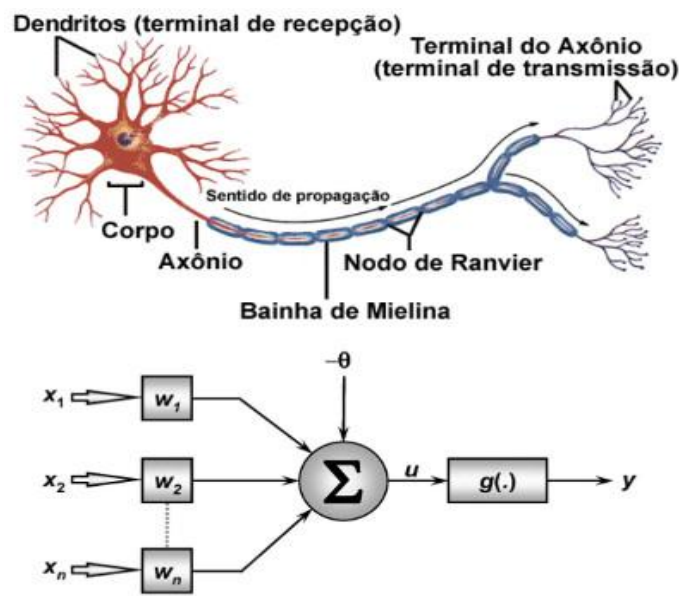

Fig. 2 - Detalhamento de neurônio artificial aplicado em redes neurais.
Uma RNA é composta por várias unidades de processamento, cujo funcionamento é bastante simples. Essas unidades geralmente conectadas por canais de comunicação que estão associados a determinado peso, e a um conjunto de treinamento. São sistemas físicos que podem adquirir, armazenar e utilizar conhecimentos experimentais, que podem alcançar uma boa performance, devido à sua densa interconexão entre os nós da rede [11]. O neurônio artificial mais simples, proposto por Mc-Culloch Pitts em 1943, é o composto de entradas (que correspondem aos dendritos), unidade de processamento (que correspondem ao corpo celular) e saídas (que correspondem aos terminais do axônio) [12].

\subsection{Descritores de Formas}

A noção de forma de objeto, apesar de intuitivamente clara pode ter muitos significados. Muitos dos objetos do mundo real são 3D, entretanto, imagem e vídeo normalmente contem projeções $2 \mathrm{D}$ dos mesmos. No caso 2D, existem duas noções de similaridade que são: baseada em região e baseada em contorno [13].

Alguns descritores são bastante simples, porém sofrem influência de operações de rotação, translação ou mudança de escala. O Perímetro é um exemplo de um descritor de borda. Um valor aproximado pode ser obtido com a contagem dos pixels ao longo da borda do objeto. Outro descritor de borda útil é o Diâmetro, que é definido como a maior distância entre dois pontos pertencentes à borda. A Transformada de Fourier baseia-se no teorema de Fourier, pelo qual toda função contínua pode ser decomposta em uma soma de funções senoidais de freqüências variadas. Para o caso discreto, que é aquele que interessa à aplicação em imagens digitais, usa-se a Transformada Discreta de Fourier (DFT) [14].

Seja uma fronteira digital de $\mathrm{N}$ pontos no plano $x y$. Considerando $\left(x_{0}, y_{0}\right),\left(x_{1}, y_{1}\right), \ldots,\left(x_{N-1}, y_{N-1}\right)$, os pontos da borda obtidos através do percurso antihorário. Para $x(k)=x k$ e $y(k)=y k$, temos então a borda expressa como:

$$
s(k)=[x(k), y(k)]
$$

Pode-se tratar cada elemento de ' $s$ ' como um número complexo:

$$
s(k)=x(k)+j y(k), \text { para } k=0,1,2, \ldots, N-1
$$

A transformada de Fourier de $s(k)$ então pode ser definida como:

$$
a(u)=\frac{1}{N} \sum_{K=0}^{N-1} s(k) \exp \left(\frac{-j 2 \pi u k}{N}\right)
$$


Suponha que apenas os $M$ primeiros coeficientes sejam utilizados. Isto é equivalente a zerar todos os valores de $a(u)$ para $u>M-1$. Então temos:

$$
S^{*}(k)=\sum_{u=0}^{M-1} a(u) \exp \left(\frac{j 2 \pi u k}{N}\right)
$$

As baixas frequências guardam informações de forma, enquanto altas frequências representam os detalhes finos. Portanto, quanto maior o valor de M utilizado, mais detalhada será a descrição da imagem.

\section{Desenvolvimento do trabalho}

O desenvolvimento presente neste projeto se baseia inicialmente, em um aplicativo mobile aonde 0 usuário/cliente reconhecerá a cor de tal objeto, baseado no algoritmo da rede neural, extraindo a informação certa e a cor desejada. O enfoque será voltado para pessoas cujo possuem o daltonismo, a dificuldade de discernir as cores verde, azul e vermelho. Com o intuito de ser aplicado na área de segurança do trabalho, portanto, as cores para segurança em locais de trabalho indicam e advertem risco. Por estas entre outras tantas razoes a questão da sinalização é de suma importância como ferramenta de prevenção.

Foi adotado a linguagem python e a API da IBM Watson, usando Visual Recognition, para reconhecimento das cores, juntamente com o framework lonic para fazer o aplicativo mobile. Devido às suas tecnologias de código aberto implícitas, o IBM Bluemix oferece flexibilidade para integrar desenvolvimento e serviços que se adaptem às suas necessidades [15].

O IBM Bluemix oferece serviços de nível básico e empresarial de que as organizações precisam para que seus aplicativos estejam prontos e disponíveis para quando e onde seus clientes mais necessitarem.

O lonic é um "poderoso framework de desenvolvimento de aplicativos nativos em HTML5 que ajuda a construir aplicativos móveis com impressão nativa, integralizando tecnologias como HTML, CSS e Javascript". Segundo lonic, este framework não foi construído com a intenção de ser executado em navegadores desktop, como o Google Chrome ou Safari, mas sim em navegadores de baixo nível, como o WebView, utilizados em aplicativos híbridos. O lonic é um framework open-source licenciado sob a licença do MIT, a qual garante que "você pode utilizar o lonic nos seus próprios projetos comerciais ou pessoais gratuitamente" [16]. A licença MIT, também chamada de licença $X$ ou de licença $X 11$, é uma licença de programas de computadores (software), criada pelo Instituto de Tecnologia de Massachusetts (MIT). Ela é uma licença permissiva utilizada tanto em software livre quanto em software proprietário. [17].

Para o desenvolvimento de aplicações utilizando lonic, não é necessário nenhum equipamento específico, assim como no desenvolvimento Android. É necessário apenas um computador com qualquer Sistema Operacional, no entanto, para o desenvolvimento, do app iOS, ainda é necessário que se possua um computador com o Sistema Operacional. O MacOS e com o Xcode. Podese utilizar qualquer IDE de acordo com as preferências do programador, não há qualquer tipo de recomendação técnica por parte da equipe ou presente na documentação do lonic. A linguagem utilizada é o JavaScript, usando ainda HTML e CSS para criação da interface gráfica. [18].

\section{Discussão dos resultados}

Ao finalizar, o usuário é capaz de identificar a referida cor interagindo com o aplicativo. A partir deste momento, o aplicativo já serve de auxílio para uma pessoa com algum tipo de discromatopsia, pois o indivíduo consegue identificar objetos ao seu redor, em tempo real, nos quais não consegue determinar a cor correta devido a sua deficiência cromática. Segue os testes obtidos:

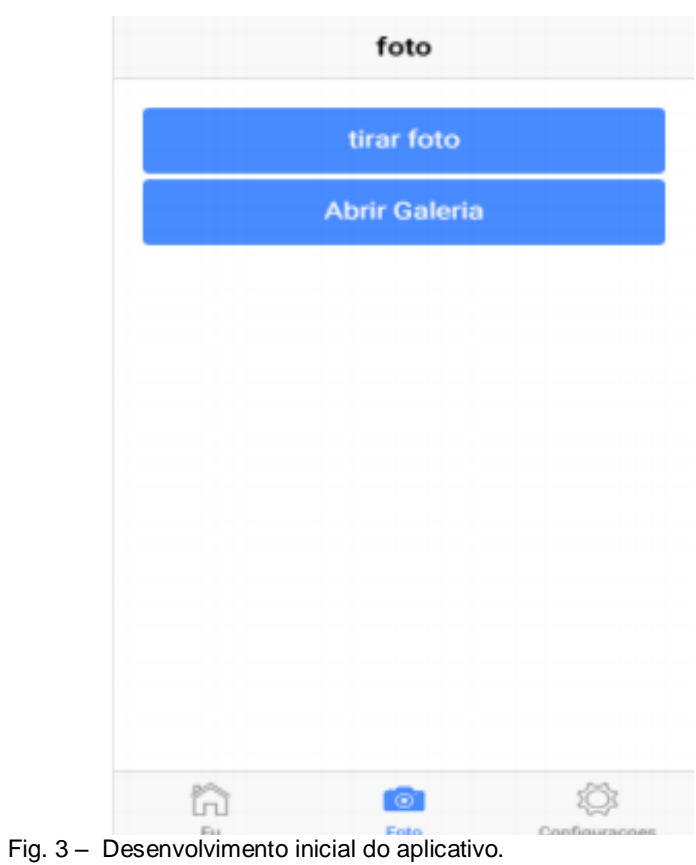




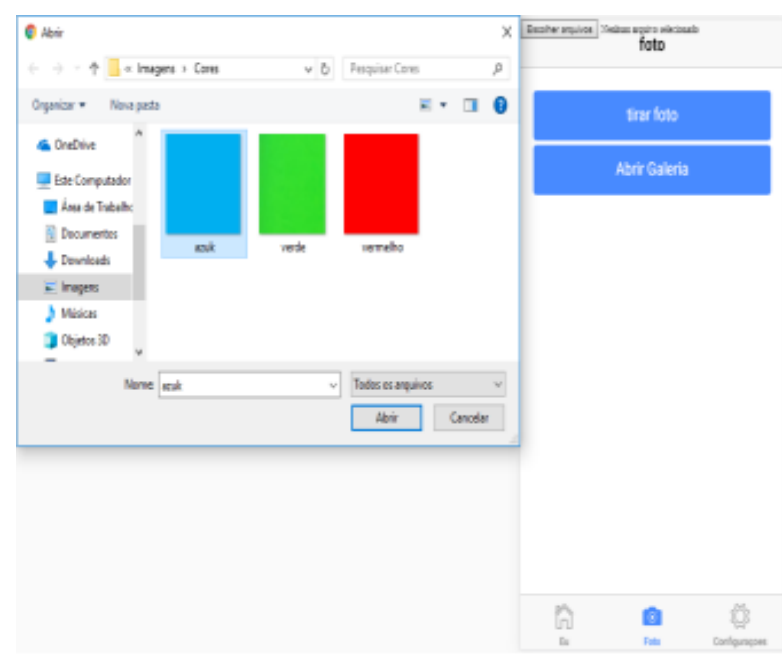

Fig. 4 - Escolhendo a cor, depois de ter clicado em galeria.

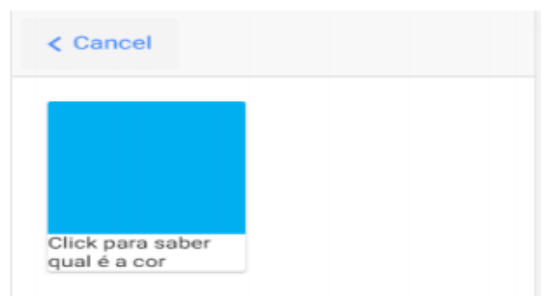

Fig. 5 - Aba para scannear a cor na Rede neural Artificial.

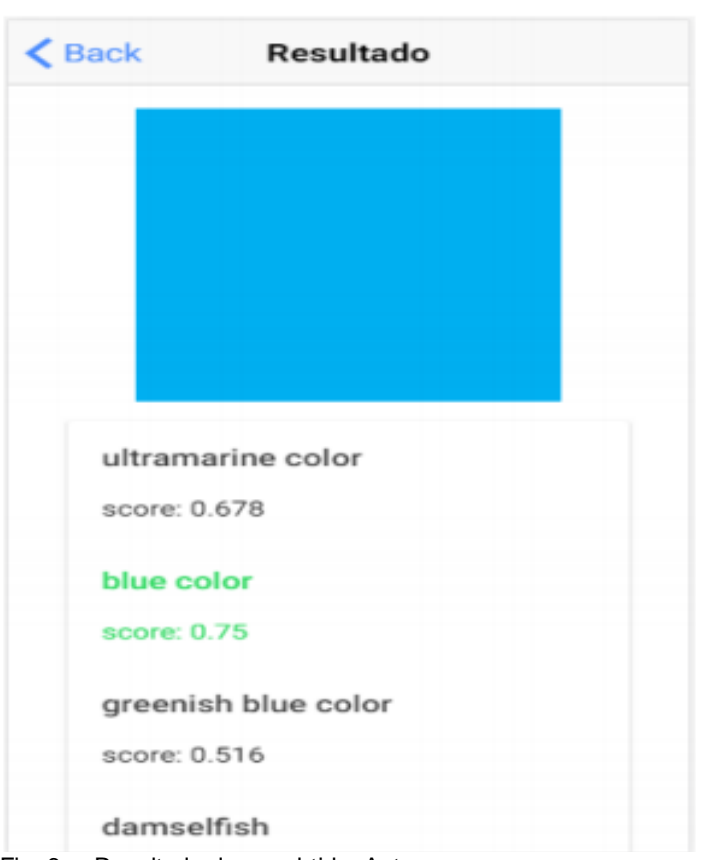

Fig. 6 - Resultado da cor obtida. Autor

\subsection{Implementação da Solução}

A implementação da ferramenta foi baseado no API da IBM junto com o framework lonic 2, no qual fez-se uso de um código importante para servir no desenvolvimento do presente trabalho:

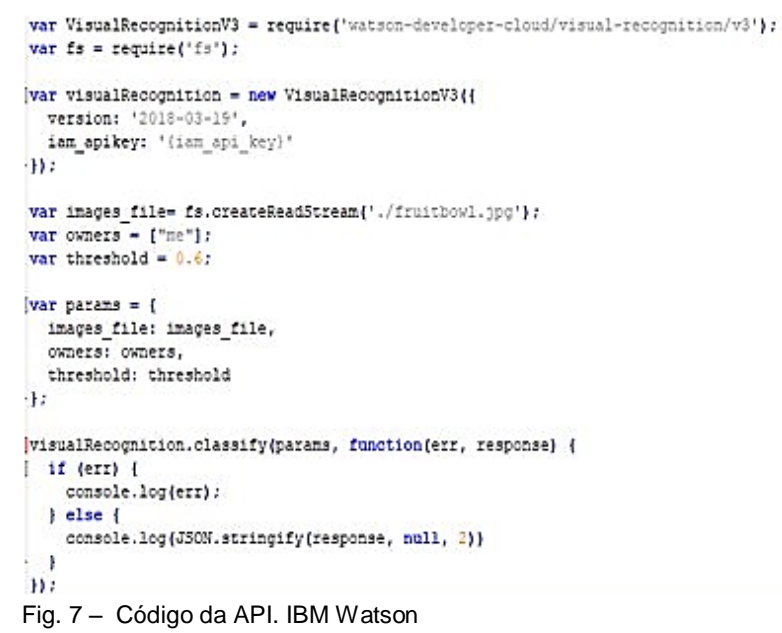

\section{Considerações finais}

$\begin{array}{ccc} & \text { O trabalho elaborado propôs uma ferramenta } \\ \text { capaz de auxiliar indivíduos que }\end{array}$ possuem discromatopsia, de forma que passem a ser capazes de reconhecer o que não está sendo distinguido da maneira correta, isto é, todos os elementos que a pessoa não consegue ou, possui certa dificuldade de distinção e percepção de uma ou mais cores. A ferramenta foi elaborada na forma de um aplicativo móvel, desenvolvido com o framework lonic3 e com o auxílio da API da IBM Recognition. Com os resultados apresentados, é possível inferir que o trabalho atingiu o objetivo geral proposto, já que foi desenvolvido o aplicativo móvel capaz de detectar e informar ao usuário daltônico as cores corretamente.

\section{Referências}

[1] PudMed Health, 2011 Color blindness [Online]. Disponível em: http://www.ncbi.nlm.nih.gov/ pubmedhealth/PMH0001997.

[2] K. S. Fu, Syntatic Pattern Recognition and Application, Englewood Cliffs, Prentice Hall, NJ, 1982

[3] Koug and Kosko,1992 "Image coding with fuzzy image segmentation". In: D Dubois and H. Prade editors, IRRR International Conference in fuzzy Systems, San Diego, pp. 213-220.

[4] Bonventi and Reali Costa,2000 "Comparação entre métodos de definição de conjuntos nebulosos de cores para a classificação de pixels". International Joint Conference IBERAMIA'2000 and SBIA'2000, Open Discussion Track Proceedings, M. C. Monard and J. S. Sichman (eds.), November 19-22, pp. 105-110 


\section{Encontro Científico de Física Aplicada}

\section{Blucher}

[5] Littman and Ritter,199S "Adaptive color segmentation: a comparison of neural and statistical methods". In: IEEE Transactions on neural networks, $8(1)$, January.

[6] S. S. Haykin. Redes Neurais : Principios e Praticas. [S.I]: Bookman,2001

[7] Gordon,1998; Bruni; Cruz,2006 sentido cromático: tipos de defeitos e testes de avaliação clínica. Arq. Bras. Oftalmol., são Paulo, v. 69, n. 5, p. 766-775

[8] Palasio, Cosmo,2009 Cores na sinalização de segurança. Disponível

[9] Vespucci,K M : Daltonicos ao Volante,2017

[10] Soares Renato : avaliaçao dos condutores portadores de discromatsopsia congenita na percepçao cromática da sinalizaçao viaria,2009

[11] FERNANDES, Anita M. da Rocha Inteligencia Artificial Noçoes Gerais, Florianopolis,Visua Books,2001

[12] Valença, Meuser : Fundamentos das Redes Neurais,2009

[13]BOBER,M. MPEG-7 Visual Shape Descriptors. IEEE Transcations on Circuits and Systems for Video
Techonology, New York, v.11, n.6, p.716-719,June 2011.

[14] RONDA, T.F; MARQUES P.M.A; FELIPE, J.C. (2008). Avaliação Comparativa de Descritores de Forma Utilizados para Caracterização e Classificação de Nódulos de Mama. Apresentado no XXI Congresso Brasileiro de Engenharia Biomédica.

[15] Reyes, A. T .2014 . O que é IBM Bluemix Disponivel em Acesso em 29.06.2017

[16] Ionic,2015 Chapter 1: All about Ionic. Disponível em: . Acesso em: 19.02.2015

[17] Institudo de Tecnologia de Massachusetts http://mit-license.org/

[18] Drifty,2016 AboutloniclonicComponents.2016.Disponívelem: Citado na página 34 